\title{
Progress on the Study and Practice of Venous Thromboembolism after Operation of Tumor
}

\author{
Shaohua Wang ${ }^{1}$, Jianli Jiang², Xiaobing Lu ${ }^{3}$ \\ ${ }^{1}$ The Second People's Hospital of Yichang, Yichang, China \\ ${ }^{2}$ The First People's Hospital of Yichang, Yichang, China \\ ${ }^{3}$ The Institute of Mechanics, Chinese Academy of Sciences, Beijing, China \\ Email:xblu@imech.ac.cn
}

How to cite this paper: Wang, S.H., Jiang, J.L. and Lu, X.B. (2020) Progress on the Study and Practice of Venous Thromboembolism after Operation of Tumor. Journal of Biosciences and Medicines, 8, 79-87. https://doi.org/10.4236/jbm.2020.88008

Received: July 14, 2020

Accepted: August 15, 2020

Published: August 18, 2020

Copyright (c) 2020 by author(s) and Scientific Research Publishing Inc. This work is licensed under the Creative Commons Attribution International License (CC BY 4.0).

http://creativecommons.org/licenses/by/4.0/

\begin{abstract}
The incidence rate of venous thromboembolism (VTE) of patients after operation is very high. Many studies on VTE have been processed. The pathogenesis, dangerous factors and assessment method of VTE are summarized in this paper. Two theories of pathogenesis, theory of Virchow's triad and theory of anoxia at the tip of venous valve, are introduced first. The main dangerous factors of VTE such as tumor, perioperative period are discussed then. The main assessment methods of VTE are introduced also. At last, some problems required to be studied deeper have been presented.
\end{abstract}

\section{Keywords}

Venous Thromboembolism, Tumor, Risk Assessment Score Model

\section{Introduction}

The incidence of malignant tumor increases significantly in the world. The venous thromboembolism (VTE) of patients after operation is $7 \%-45 \%$ [1], which is a great threat to the patients' life. VTE is that the blood clots in the vein due to complex pathophysiological process and so blocks the blood reflux. Thromboembolism can be divided into arterial and venous thromboembolism. The ischemic heart disease and ischemic stroke are two main arterial thromboembolic diseases. Deep venous thrombosis (DVT) and pulmonary thrombus embolism (PTE) are two typical VTE diseases [2] [3].

If VTE is not found and cured in time, acute thrombus exfoliation with pulmonary embolism and even death can happen because its formation is generally insidious [4]. For example, the number of deaths due to thrombus exfoliation in 2010 is over $25 \%$ of the total number in the world [5] according to the statistics. 


\section{Pathogenesis of VTE}

VTE is the third type of vascular disease second only to the ischemic heart disease and ischemic stroke. It brings large burden to the global economy and health.

There are two kinds of theories on the pathogenesis of VTE. One is the theory of Virchow's triad, the other is the theory of valve cusp hypoxia thesis [6].

\subsection{Theory of Virchow's Triad}

Virchow's triad, named after the German physician Rudolf Virchow, means blood stasis, hypercoagulable state and endothelial injury. The core of the theory of Virchow's triad is that the dynamic equilibrium among the coagulation system, anticoagulation system and fibrinolysis system is destroyed by any of the three factors (blood stasis, hypercoagulable state and endothelial injury). The lost of equilibrium causes the formation of VTE. Under normal conditions, contraction of calf muscles plays a role in "muscle pump" with the help of venous valve [7]. The venous valve acts as a check valve which makes the blood flow toward the heart. Under the combined action of "muscle pump" and "check valve", the blood in the veins can only flow toward the heart and meanwhile disperse the cells and other materials related with blood clotting [8]. However, if one has to lie in bed for a long period or reduce the movement of legs due to some reasons such as coma, lower extremity dyskinesia, leakage of cerebrospinal, the damage of the structure and function of venous valve etc. the actions of "muscle pump" and "check valve" will disappear and the blood speed will decrease. The blood can appear to be in hypercoagulation state in local region due to the accumulation of a lot of cells and coagulation factors. In normal physiological state, vascular endothelial cells play a role in anticoagulation and prevent the thrombosis. However, if vascular endothelial cells are damaged widely, the tissue factor and collagen will expose directly in the circulating blood. A mounts of blood platelets will adhere and assemble near the damaged vascular endothelial cells to cause coagulation cascade. Meanwhile, the damaged vascular endothelial cells can synthetize plasminogen activator inhibitor to restrain the fibrinolysis system. All the above factors can cause thrombosis.

\subsection{Theory of Anoxia at the Tip of Venous Valve}

The venous valve pocket (VVP) is the lacuna enclosed by the venous valves and the wall of vein. The volume of VVP can change obviously with the opening and closing of venous valves, which can change the flow dynamics of the blood near VVP. It is the core of this theory.

When the venous blood flows through the tip of venous valve, a small part of blood enters into VVP to form very slow vortex and stays in VVP for quite a long period. In normal conditions, "muscle pump" can produce discontinuous pulse flow besides the power for venous return which makes the blood in VVP renewed fast by the opening and closing of the vein valve [9]. If the function of 
the "muscle pump" is failed due to coma or paralysis of patients, the vein valve cannot open and close with the changes of pressure. Then venous valve will be in continuous semi-opening mode. The deep vein blood flow of lower extremity becomes to be non-pulse flow, which will reduce the exchange of blood between VVP and vena cava. In other words, the change of the function of vein valve and flow characteristics of blood reduces the movement of the vein valve. The blood has to stay in VVP for longer time, which can cause the blood platelet and leukocyte collected in the vortex. Then oxygen in VVP is consumed and so the blood oxygen concentration decreases gradually. VTE happens under the above factors.

In general, theory of Virchow explains the occurrence by the destruction of the dynamic equilibrium among the coagulation system, anticoagulation system and fibrinolysis system. The three factors of this theory can perfectly relate the clinical dangerous factors, but the mechanism of VVP cannot be explained well by this theory. The theory of anoxia at the tip of venous valve presents a detailed single mode for the occurrence of VTE, but it cannot explain the formation mechanism of VTE in deep veins which have no venous valve. Though someone attempts to explain the occurrence of VTE by combining these two theories, but the combination is not widely accepted. Therefore, more complete practical theory should be presented to reveal the formation mechanism of VTE.

\section{Factors Affected VTE}

The dangerous factors caused VTE can be divided into three types [10]: factors related with tumor directly, factors related with patients and factors related with treatment. Generally, tumor patients have these three types of dangerous factors at the same time.

\subsection{Danger Factors Related with Tumor}

The main factors causing postoperative VTE vary with tumor types. That means, the prevention measures must vary with the type of tumor.

The common dangerous factors related with tumors of nervous system include age, obesity, long term operation, postoperative coma, paralysis of limbs, postoperative endotracheal intubation over 48 hours, long term bed rest after operation, use of dehydrating agents, oral contraceptives, use of glucocorticoid, period of pregnancy or puerperium, infection, history of thrombosis etc. [6] [11].

The dangerous factors related with VTE after operation of breast tumor include age, pathological grading, time of operation, time of activating partial thromboplastin etc. The dangerous factors related with VTE after operation of ovarian tumor are age and total of platelet.

For patients with gynecologic tumor, the main dangerous factors related with VTE are age, tumor staging, degree of tumor differentiation, lymph node metastasis and complication (hypertension, diabetes) [12] [13]. It is shown that the 
risk occurring VTE at stage IV is 1.452 times at other stages. Obesity and ethnic differences are also the dangerous factors. The risk of the patient having family history of VTE is 3.44 times that without family history. The risk of obesity is 1.42 times of the non-obesity [10] [14].

Prevention measures at perioperative period of gynecologic tumor can effectively reduce the risk of VTE and other complications [15] [16]. Once VTE occurs to a tumor patient, he/she has to prolong the period staying in the hospital and affects the treatment of tumor. The mortality will increase [13] [17].

The dangerous factors related with VTE of esophageal carcinoma during Perioperative period are as follows: age over 65, distant metastasis, history of VTE, central venous catheterization etc. Patients having any factor of these must be given anticoagulant therapy preventively [18].

For inpatients of internal medicine-oncology, history of VTE, lying in bed over 3 days, platelet count over $350 \times 109 / \mathrm{L}$, D-dimer over $0.55 \mathrm{mg} / \mathrm{L}$, tumor stage are the main dangerous factors of VTE [19].

Dangerous factors related with patients' agedness, obesity, leukocytosis before chemotherapy, anaemia, thrombocytopenia, catheter ectopia during catheterization, catheter blockage, catheter related infection are the common main factors of VTE relate with tumor [20]. Infection, bad environment for treatment and living increase the risk of VTE [21]. However, it is shown that the sexuality, history of hypertension, glycuresis, smoking and drinking are not related with VTE [22].

If patients of primary liver tumor have long term smoking history, family history of VTE, great vein injury, or the blood capillary are invaded, or suffer from portal vein tumor thrombus, he/she is easy to get VTE [23] [24].

\subsection{Dangerous Factors Related with Perioperative Period}

The antineoplastic therapy such as operation, chemotherapy, endocrine therapy, antiangiogenic agents, central venous catheterization will increase the risk of VTE [25] [26].

The risk of VTE is higher with the rise of tumor staging during chemotherapy before operation. The risk of concurrent VTE is the highest during the operation of abdominal tumor, nervous system tumor and tumor of bone and joint. The risk of VTE increases with the extension of time. Intraoperative blood transfusion also affects the occurrence of VTE. The blood transfusion can damage the equilibrium of coagulation factors, which can induce postoperative VTE by combining with the hypercoagulable state inside the patients of tumors. If the surgical area is the central nervous system, or spine, pelvic floor, lower limb joint, or the lower extremity vessels, VTE is easier to happen than in other areas.

Operation types causing postoperative VTE are often as follows: thrombectomy of lower extremity artery or endarterectomy, total hip replacement or revision of hip joint, resection of intracranial nervous system, injury and biopsy, hemiarthroplasty etc. Operation mode can also cause VTE. For example, there is 
pneumoperitoneum in laparoscopic surgery and robotic surgery, so the venous return pressure of lower extremity increases. Accordingly, the possibility of VTE increases. During limb rehabilitation therapy, the fixation time of local lower limb will prolong, which increases the incidence of complications of VTE. If there exist iatrogenic puncture, pressure dressing during perioperative period, the vein wall can be damaged. This is another factor related with VTE during operation [27]. During hepatectomy, reoperation or multiple operations are the strong dangerous factors causing VTE. The risk is 6.21 times of those with only one operation. The operation time prolongs every hour, the risk to cause VTE increases 1.27 times [24]. The use of intermittent pneumatic pump after operation can reduce the risk of VTE to $22.7 \%$.

Totally, the common dangerous factors with operation are as follows: old, sepsis within 1 month, local movement disorder of lower limbs, iliac vein compression, congenital or secondary stenosis of inferior vena cava, congestive heart failure, long time lying in bed after operation, obesity, acute myocardial infarction, cerebral apoplexy, ethnic group, diabetes mellitus, hyperlipidemia, use of coagulant drugs in perioperative period, insufficient circulation, long term oral contraceptives, hormone replacement therapy, use of narcotic drugs, history of DVT or PET, family history of VTE, lupus anticoagulant positive, elevated serum homocysteine, heparin induced thrombocytopenia, positive of prothrombin, severe hypoproteinemia. Smoking is thought to be a co-factor of other factors $[5]$.

\section{Methods of Risk Assessment of VTE}

At present, in clinical practice, many risk assessment score models have been built for assessment of VTE, such as Caprini risk assessment score model, Rogers risk assessment score model, Khorana risk assessment score model, Padua risk assessment score model etc.

Caprini risk assessment score model: In 2005, Caprini presented a model for VTE assessment based on the statistics of patients in surgical department, cardiovascular department, urology (called Caprini Risk Assessment Score Model) [28]. The model is often used in the screening and diagnosis. However, Caprini model still exists some defects in risk assessment of VTE for tumor patients. The main reason is that the model is based on surgical patients but not tumor patients. The risk of VTE varied with treatment in practice. That means, the applicability of Caprini model in risk assessment of VTE for tumor patients needs still to be investigated. Meanwhile, the factors considered in Caprini model are too many to collect clinical data. These defects limit the application of Caprini model in the risk assessment for tumor patients.

Rogers risk assessment score model: the model is presented by Rogers in 2007 [29] and considers the common dangerous factors, surgical types except endocrine surgery, classification of physical condition by American society of anesthesiologists and work RUV (related unit of value). However, this model is not widely used because there are too many and complex factors in this model. 
Khorana risk assessment score model: Khorana presented an assessment score model for the risk evaluation of tumor patients in 2008 [13]. Khorana risk assessment model is thought to be the best one for the VTE risk evaluation of patients in oncology department. But the recent study shows that high Khorana score cannot predict VTE.

The Padua assessment score model: In 2010, Padua University presented the Padua assessment score model based on inpatients in internal medicine [16]. The Padua assessment score model is mainly for the internal medicine patients in general hospitals and so most of the factors in this model do not often exist in patients of oncology department. In addition, The Padua assessment score model needs more data for validation.

Autar assessment score model: Autar model is presented by Autar in De Montfort University. This model contains age, body mass index, activity ability, trauma risk, high risk disease, special risk, surgery. The practice shows that this model is suitable for the risk assessment of VTE, especially for spinal surgery, hip replacement etc. [30].

Wells assessment score model: It is shown that the Wells model has high practicability. This model considers the clinical symptoms and signs of VTE, heart rate, time lying in bed, previous operation within 1 month, history of VTE, hemoptysis, tumor [31].

There are many risk factors of VTE related tumor should be considered [19]. Previous assessment score models have only limited generality. In clinical practices, anyone of the presented models cannot be used in the risk assessment of VTE in different stage of treatment or different tumors. Further study is required to develop models of VTE risk assessment with more adaptability.

\section{Conclusions}

VTE of patients after tumor operation is a great threat to the patients' life. Understanding of the formation mechanism and prevention methods is very important.

The formation mechanism of VTE is summarized in the first part. Two types of theories, the theory of Virchow's triad and the theory of valve cusp hypoxia thesis, are introduced carefully. The base of the pathogenesis in these two theories is explained. The main reason of VTE is due to the clotting of blood. However, anyone of these two theories cannot explain the formation mechanism of all kinds of VTE.

The dangerous factors caused VTE are divided into three types and summarized: factors related with tumor directly, with patients and with treatment respectively. Generally, these three types of factors are existed in patients of tumor after surgery. The possibility changes with the factors that patients have.

In the future, the formation mechanism of VTE needs to study further to present better practical preventing methods. More practical assessment methods should be built either. 


\section{Conflicts of Interest}

The authors declare no conflicts of interest regarding the publication of this paper.

\section{References}

[1] Cai, Q. and Zhao, S.P. (2016) The High Risk Factors of Postoperative Venous Thromboembolism of Gynecological Cancer Patients and Prevention Measures. China Continuing Medical Education, 30, 74-75. (In Chinese)

[2] Line, B.R. (2001) Pathophysiology and Diagnosis of Deep Venous Thrombosis. Seminars in Nuclear Medicine, 31, 90-101. https://doi.org/10.1053/snuc.2001.21406

[3] Olaf, M. and Cooney, R. (2017) Deep Venous Thrombosis. Emergency Medicine Clinics of North America, 35, 743-770. https://doi.org/10.1016/j.emc.2017.06.003

[4] Cerrato, D., Ariano, C. and Fiacchino, F. (1978) Deep Vein Thrombosis and Low-Dose Heparin Prophylaxis in Neurosurgical Patients. Journal of Neurosurgery, 49, 378-381. https://doi.org/10.3171/jns.1978.49.3.0378

[5] Chen, J.D. (2018) Trends of Cancer Incidence and Mortality in China. Journal of Zunyi Medical University, 41, 653-662. (In Chinese)

[6] Cao, Y.K. (2019) Study on the Risk Factors of Lower Extremity Deep Venous Thrombosis during Postoperative Period in Patients after Brain Neoplasms Resection. Thesis for Master Degree, Jilin University, Changchun. (In Chinese)

[7] Merli, G.J. (2008) Pathophysiology of Venous Thrombosis and the Diagnosis of Deep Vein Thrombosis-Pulmonary Embolism in the Elderly. Cardiology Clinics, 26, 203-219. https://doi.org/10.1016/j.ccl.2007.12.013

[8] Merli, G.J. (2006) Pathophysiology of Venous Thrombosis, Thrombophilia, and the Diagnosis of Deep Vein Thrombosis-Pulmonary Embolism in the Elderly. Clinics in Geriatric Medicine, 22, 75-92. https://doi.org/10.1016/j.cger.2005.09.012

[9] Karino, T. and Motomiya, M. (1984) Flow through a Venous Valve and Its Implication for Thrombus Formation. Thrombosis Research, 3, 245-257. https://doi.org/10.1016/0049-3848(84)90224-X

[10] Algattas, H., Kimmell, K.T., Vates, G.E., et al. (2015) Analysis of Venous Thromboembolism Risk in Patients Undergoing Craniotomy. World Neurosurgery, 84, 1372-1379. https://doi.org/10.1016/j.wneu.2015.06.033

[11] Xiong, A.L. (2018) Analysis of Risk Factors for Venous Thrombosis after Gynecology Malignant Tumor Surgery. Thesis for Master Degree, Guangxi Medical University, Nanning. (In Chinese)

[12] Sundbøll, J., Veres, K., Horváth-Puhó, E., et al. (2018) Risk and Prognosis of Cancer after Lower Limb Arterial Thrombosis. Circulation, 138, 669-677. https://doi.org/10.1161/CIRCULATIONAHA.117.032617

[13] Khorana, A.A., Kuderer, N.M., Culakova, E., et al. (2008) Development and Validation of a Predictive Model for Chemotherapy-Associated Thrombosis. Blood, 111, 4902-4907. https://doi.org/10.1182/blood-2007-10-116327

[14] Agnelli, G., Gussoni, G., Bianchini, C., et al. (2009) Nadroparin for the Prevention of Thromboembolic Events in Ambulatory Patients with Metastatic or Locally Advanced Solid Cancer Receiving Chemotherapy: A Randomised, Placebo-Controlled, Double-Blind study. The Lancet Oncology, 10, 943-949. https://doi.org/10.1016/S1470-2045(09)70232-3

[15] Chen, Y. and Wang, D.H. (2019) Analysis of Risk Factors of Venous Thromboem- 
bolism after Three Major Gynecological Malignant Tumors Surgery. Journal of Zunyi Medical University, 42, 698-701. (In Chinese)

[16] You, Y.Q. (2019) Effect of Preventive Nursing on Preventing Deep Venous Thrombosis of Lower Limbs in Perioperative Period of Gynecological Tumor Operation. Guide of China Medicine, 17, 233-234. (In Chinese)

[17] Barbar, S., Noventa, F., Rossetto, V., et al. (2010) A Risk Assessment Model for the Dentification of Hospitalized Medical Patients at Risk for Venous Thromboembolism: The Padua Assessment Score. Journal of Thrombosis and Haemostasis, 8, 2450-2457. https://doi.org/10.1111/j.1538-7836.2010.04044.x

[18] Yasenjiang, M. and Zhang, L.W. (2020) Analysis of Perioperative Venous Thrombosis and Risk Factors in Patients with Esophageal Cancer. Chinese and Foreign Medical Research, 18, 129-131. (In Chinese)

[19] Men, X.Q., Li, H.M., Dong, Q., et al. (2019) Risk Factors of Venous Thromboembolism and Evaluation of Existing Risk Assessment Models for Predicting Venous Thromboembolism in Medical Oncology Inpatients. Chinese Journal of Practical Internal Medicine, 39, 694-697. (In Chinese)

[20] Ageno, W., Squizzato, A., Garcia, D., et al. (2006) Epidemiology and Risk Factors of Venous Thromboembolism. Seminars in Thrombosis and Hemostasis, 32, 651-658. https://doi.org/10.1055/s-2006-951293

[21] Rogers, M.A., Levine, D.A., Blumberg, N., et al. (2012) Triggers of Hospitalization for Venous Thromboembolism. Circulation, 125, 2092-2099. https://doi.org/10.1161/CIRCULATIONAHA.111.084467

[22] Xu, T.F. (2019) Clinical Analysis of Perioperative Symptomatic VTE in Hepatic Resection of Primary Liver Cancer and Improvement and Application of Caprini Risk Assessment Model. Thesis for PhD, Guangxi Medical University, Nanning. (In Chinese)

[23] He, Y.Y. (2017) Establishment of Risk Assessment Model for PICC-Related Upper Extremity Venous Thrombosis in Patients with Breast Cancer Chemotherapy. Thesis for Master Degree, Bengbu Medical College, Bengbu. (In Chinese)

[24] Zhao, Y.N. (2019) Clinical Analysis of Perioperative Symptomatic VTE in Hepatic Resection of Primary Liver Cancer and Improvement and Application of Caprini Risk Assessment Model. Thesis for PhD, Guangxi Medical University, Nanning. (In Chinese)

[25] Verso, M. and Agnelli, G. (2003) Venous Thromboembolism Associated with Long-Term Use of Central Venous Catheters in Cancer Patients. Journal of Clinical Oncology, 21, 3665-3675. https://doi.org/10.1200/JCO.2003.08.008

[26] Men, X.Q. (2019) The Study of Establishment and Evaluation of New Risk Assessment Model for Predicting Venous Thromboembolism in Medical Oncology Inpatients. Thesis for Master Degree, China Medical University, Shenyang. (In Chinese)

[27] Guo, W.C., Yu, B. and Shi, W.H. (2020) Risk Factors and Progress in Diagnosis and Treatment of Surgical Inpatients Complicated with Lower Extremity Deep Vein Thrombosis. Chinese Journal of Practical Surgery, 40, 594-598. (In Chinese)

[28] Caprini, J.A. (2005) Thrombosis Risk Assessment as a Guide to Quality Patient Care. Disease-a-Month: DM, 51, 70-78. https://doi.org/10.1016/j.disamonth.2005.02.003

[29] Rogers, S.O., Kilaru, R.K., Hosokawa, P., et al. (2007) Multivariable Predictors of Postoperative Venous Thromboembolic Events after General and Vascular Surgery: Results from the Patient Safety in Surgery Study. Journal of the American College of Surgeons, 204, 1211-1221. https://doi.org/10.1016/j.jamcollsurg.2007.02.072 
[30] Zhang, X.D., Gong, P. and Liu, J.H. (2018) Comparison between Autar and Padua Risk Assessment Models for Cancer Patients with Venous Thromboembolism. Chinese Journal of Surgical Oncology, 10, 237-240. (In Chinese)

[31] Lim, W., Le, G.G., Bates, S.M., et al. (2018) American Society of Hematology 2018 Guidelines for Management of Venous Thromboembolism: Diagnosis of Venous of Thromboembolism. Blood Advances, 2, 3226-3256.

https://doi.org/10.1182/bloodadvances.2018024828 